\title{
Functional Somatic Syndromes: Emerging Biomedical Models and Traditional Chinese Medicine
}

\author{
Steven Tan, Kirsten Tillisch and Emeran Mayer \\ Center for Neurovisceral Sciences and Women's Health, Department of Medicine, Physiology and Psychiatry, David \\ Geffen School of Medicine, UCLA
}

\begin{abstract}
The so-called functional somatic syndromes comprise a group of disorders that are primarily symptom-based, multisystemic in presentation and probably involve alterations in mind-brain-body interactions. The emerging neurobiological models of allostasis/allostatic load and of the emotional motor system show striking similarities with concepts used by Traditional Chinese Medicine (TCM) to understand the functional somatic disorders and their underlying pathogenesis. These models incorporate a macroscopic perspective, accounting for the toll of acute and chronic traumas, physical and emotional stressors and the complex interactions between the mind, brain and body. The convergence of these biomedical models with the ancient paradigm of TCM may provide a new insight into scientifically verifiable diagnostic and therapeutic approaches for these common disorders.
\end{abstract}

Keywords: functional somatic syndromes - allostasis - emotional motor system - stress - mind-brainbody interactions

\section{Introduction}

The functional somatic syndromes comprise a large group of symptom-based disorders that are poorly understood and inadequately treated. Nevertheless, they have a major impact on the health-related quality of life (HRQoL) of affected patients. These disorders include (but are not limited to) fibromyalgia, myofascial pain syndromes, chronic headaches, temporomandibular disorder, chronic fatigue syndrome, irritable bowel syndrome (IBS), interstitial cystitis and pelvic pain syndromes. Reliable and specific biological disease markers to assist in diagnosis have not yet been identified (14). As a result, each of these syndromes is currently defined by separate symptom-based diagnostic criteria (4-9). Although certain syndromes have presentations with symptoms predominant in one organ system (e.g., bowel symptoms in IBS), patients almost universally present with significant symptomatology across multiple organ systems $(2,4,6,10)$. Another feature that is common to these disorders is a strong history of physical and/or emotional stressors and concurrent mood disturbances $(1,7,11)$. Since few conventional medications have shown satisfactory results, several patients have turned to complementary and alternative modalities for relief $(12,13)$.

For reprints and all correspondence: Emeran A. Mayer, Center for Neurovisceral Sciences and Women's Health, VA GLAHS, CURE Bldg. 115, Rm. 223, 11301 Wilshire Blvd., Los Angeles, California 90073, USA.

Tel: 310-312-9276 Fax: 310-794-2864 E-mail: emayer@ucla.edu
Two emerging biomedical models with the potential to further our understanding of the functional somatic syndromes are allostasis/allostatic load and the emotional motor system (EMS) (14-18). These models explore the complicated interactions of physical and emotional stressors in the genesis of symptoms and diseases. Similarly, in the ancient paradigm of Traditional Chinese Medicine (TCM), physical and emotional states are inextricably linked with maintaining health or precipitating illness (19-21). In this paper, we discuss how the convergence of these biomedical models with the ancient healing tradition of TCM may provide novel perspectives in understanding these challenging and elusive disorders.

\section{The Challenge of Functional Somatic Syndromes}

Functional somatic syndromes pose a challenge to clinicians for several reasons.

\section{Functional Versus Organic}

Current biomedical thinking emphasizes identification of specific diseases to explain symptoms over analysis of general symptom patterns (4-6). Diseases are usually defined by specific pathophysiological processes using generally agreed upon biological markers. In the case of functional somatic syndromes, the etiology and pathophysiology are yet to be identified, and no reliable diagnostic markers are currently available. Hence, diagnosis of these syndromes is made on the basis of symptom-based criteria. The lack of detectable 
physiological or anatomical abnormalities often diminishes the relevance, or even questions, the actual existence of such symptoms and syndromes (22-27). This implication is seen in the general designation of these disorders as 'functional' as opposed to 'organic' $(4-6,10)$.

\section{Multisystemic Manifestations}

Although affected patients frequently present with predominant symptom features pertaining to one organ system at the time of consultation, the majority has multisystemic symptomatology when assessed comprehensively. The presence of multiple symptoms involving pain, discomfort and decrease of vital functions is believed to result in a significant impairment of HRQoL of affected patients $(28,29)$. Symptom clusters, which defy a unifying disease process defined at the organ, cellular, genetic and molecular levels, are challenging to understand with our current state of medical knowledge and technology $(5,6)$. This may explain why several different subspecialties apply their own subspecialty-biased conceptualization and symptom criteria to the same group of disorders. This process creates the false appearance of multiple, separate diseases that require different, peripherally targeted treatments, even though the relevant peripheral targets remain elusive.

Emerging approaches that incorporate a broader 'holistic' view of these disorders may be more appropriate. Specifically, the authors of this review and other researchers have proposed that the multiple, and seemingly disparate symptoms of affected patients represent more general patterns of dysregulation of mind-brain-body interactions $(2,10,30-32)$. This concept is supported by the failure of medications aimed at specific peripheral targets (e.g., normalization of altered bowel movements in IBS) to substantially improve overall symptoms and HRQoL measures. In contrast, treatment of these disorders with agents such as tricyclic anti-depressants and other centrally-acting medications has achieved greater success $(3,29,33-35)$.

\section{Stress Sensitivity}

Another challenge to clinicians is that several functional disorders are strongly associated with a history of certain types of stress, and frequently overlap with disorders of mood and affect. This association, combined with the lack of detectable abnormal diagnostic tests, has often led clinicians to label these symptoms as 'psychosomatic,' 'somatoform' and 'hypochondriacal' $(3,4,7,31)$. Modern medicine has only recently begun to systematically explore the association between emotional and cognitive states and physiological changes. Current research, including observational and epidemiological studies, have shown associations between certain types of stressors (in particular, sustained, severe and uncontrollable stressors) and adverse clinical outcomes in diseases involving almost every organ system $(14,15,18)$. The specific physiological mechanisms by which mind-brain-body interactions actively maintain health, and the manner in which a dysregulation of these interactions can result in chronic disease are rapidly being identified (14). The authors believe that a better characterization of the connections between stress, mind and body will probably play a crucial role not only in a better understanding of health and disease, but in shifting the focus from arbitrarily defined symptom criteria to a physiologically-based understanding of functional somatic syndromes.

\section{Allostasis/Allostatic Load and the EMS as Biomedical Models to Explain Functional Somatic Syndromes}

Two closely related biomedical models that show promise in providing a scientific, biomedical basis for understanding the multisystemic presentations of functional somatic syndromes are allostasis/allostatic load (36) and the EMS (16). Together, these two models provide a useful framework to explain multiple symptom presentations, central dysregulations and the association of symptoms with psychosocial factors.

\section{Allostasis and Allostatic Load}

Allostasis refers to the process by which our bodies maintain homeostasis in response to environmental change or stressors. Key homeostatic mechanisms through which allostasis works include ascending monoaminergic systems (including the serotonergic, noradrenergic and cholinergic pathways), the hypothalamic-pituitary-adrenal (HPA) axis, endogenous pain modulation networks and autonomic and skeletomotor pathways. Allostatic responses to limited or acute changes in the environment are generally adaptive in nature. However, persistent stressors and/or chronic illness result in maladaptive responses reflected by sustained hyperactivity or ineffectiveness of allostatic mediators. This breakdown of the system and the consequent inability of the organism to maintain homeostasis ultimately results in a reduction of HRQoL and well-being of affected individuals (30). The damage that occurs within the organism when the allostatic response functions improperly is referred to as the allostatic load. This allostatic load can result in several common medical syndromes, ranging from ischemic heart disease and the metabolic syndrome to symptom complexes referred to as functional somatic syndromes $(14,15)$.

\section{EMS}

The EMS is central to regulating mind-brain-body interactions (Fig. 1). It explains the manner in which perturbations of the organism's homeostasis initiate stereotypic events in central regulating pathways, resulting in distinct, emotionspecific patterns of changes in the body (body maps). These bodily changes are highly adaptive to the organism's response to the environment, but are not necessarily associated with conscious perception of distinct emotional feelings (37). Neuroanatomically, the EMS refers to a set of parallel, efferent pathways that regulate the somatic, pain modulatory, auto- 


\section{Altered Responsiveness of EMS to External and Internal Stressors Produce IBS Symptom Complex}

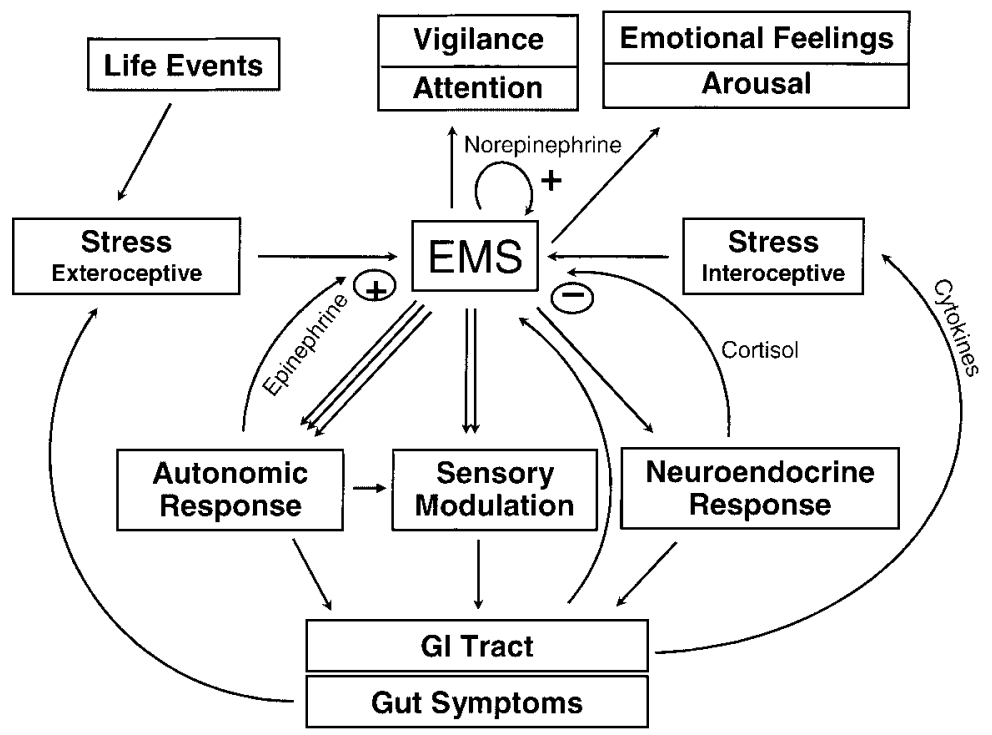

Figure 1. The Emotional Motor System. The EMS refers to a parallel set of outputs from limbic and paralimbic circuits, which generate distinct patterns of body functions ('body map') associated with specific emotions (fear, anger, joy, etc). These outputs occur in the form of autonomic nervous system responses, sensory modulations and HPA axis responses. Feedback from the body to the EMS in the form of afferent nerve signals and neuroendocrine signals modify EMS responses. Ascending outputs to cortical regions of the brain generate patterns of vigilance, arousal and attention. The conscious perception of emotional feelings may or may not be associated with activities of the EMS (modified from Mayer et al. 2001) (44).

nomic and neuroendocrine responses of the body to internal and external perturbations. The mind and body outputs of these efferent pathways, in turn, serve as feedback inputs into EMS circuits, explaining how the mind and body interactions are interlinked and mutually dependent. Vulnerability factors for altered responsiveness of subsystems of the EMS include genetic factors, early life experiences and severe life threatening stressors (Fig. 2) $(16,17,30)$.

\section{The TCM Perspective on Functional Somatic Syndromes}

In TCM, health is defined as a state of optimal balance between opposing processes in the body, referred to as yin and yang. Individuals have an underlying genetically determined constitution, referred to as jing, which reflects the innate ability of the individual to maintain balance and health. This constitution is either strengthened or weakened throughout an individual's lifetime, depending on life style,

\begin{tabular}{|c|c|c|c|}
\hline Prenatal & Early Li & & Adult \\
\hline \multicolumn{2}{|c|}{ Vulnerability Factors } & Trigger Factors & Perpetuating Factors \\
\hline
\end{tabular}

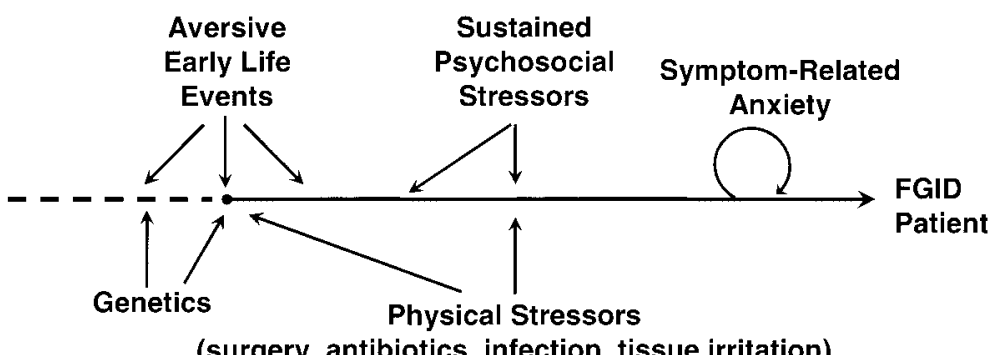

Figure 2. Interaction of genetic factors with early life events to generate vulnerabilities for stress-sensitive disorders. Genetic predisposition (presumably multigenetic traits) interacts with prenatal and early life stressors to program the stress responsiveness (or resilience towards stress) of the adult organism. This enhanced stress responsiveness pertains to both psychological and physical stressors. Perpetuating factors that play a role in the chronicity of symptoms include symptom-related fears and anxiety (modified from Mayer and Collins, 2002) (45). 
physical stressors, environmental exposure and emotional stressors. In contrast to conventional medicine, TCM emphasizes that emotional well-being and physical health are inextricably linked. In fact, TCM has elaborate theories on the differential association of distinct emotional patterns with different bodily processes (19-21,35).

When exposed to a stressor, the system attempts to maintain balance by engaging a series of responses through mediators described in ancient medical literature such as qi, blood and essence, to name a few. Imbalances result when these mechanisms fail to maintain a balance between yin processes and yang processes. This underlying imbalance systemically disrupts physiological processes, which accounts for a constellation of symptom manifestations (19-21,35). Initial responses to an acute stressor via these physiological mediators are generally adaptive in nature. However, given sustained stressors or an acute, severe stressor in a weakened system, these adaptive processes may be severely compromised or permanently disabled. This results in an exhaustion of homeostatic reserves manifesting as persistent, unresponsive and chronic symptoms commonly encountered in functional somatic syndromes.

From the TCM perspective, symptoms represent the clinical manifestation of a broader underlying pattern of dysregulation. As a result, a single presenting symptom is rarely studied in isolation. Instead, the presenting symptom is assessed in the context of concurrent symptoms, as well as symptom modifiers. TCM practitioners use this macroscopic symptom assessment with other clinical observations such as tongue and pulse diagnosis to identify the most probable pattern of dysregulation. The pattern diagnosis is then used to formulate a treatment plan using acupuncture and herbal medicine (19-21,35). A natural consequence of this clinical approach is that several patients presenting with the same chief complaint and Western disease diagnosis (e.g., constipation-predominant IBS, interstitial cystitis, heartburn) may each receive a different TCM pattern diagnosis. Patients with the same Western diagnosis may receive different treatments with acupuncture and herbs. The awareness that different subgroups of patients present with the same chief complaint reflects our increasing understanding of the existence of different subgroups of patients within each functional somatic syndrome, which may show differential responses to different therapeutic interventions.

\section{The Convergence of Biomedical Models and TCM}

Although their derivations were in different millennia, the modern biomedical models of allostasis/allostatic load and the EMS share striking similarities with the ancient paradigm of TCM. Their convergence conceptualizes a possible explanation for the genesis of many of the functional somatic syndromes that currently confound and defy conventional approaches.
Unlike conventional medicine's emphasis on physical stressors, the allostatic load model places equal emphasis on the significance of emotional and mental stressors as having serious detrimental effects on the body. The TCM observation that psychological stressors lead to physical symptoms and vice versa can be modernized through allostasis/allostatic load and the EMS. They not only describe the physiological output resulting from emotional stressors but also describe a modifiable gain system affected by physiological input from the periphery.

Both stress the fundamental importance of homeostasis in the body. In fact, in modern Western and TCM paradigms, the basic goal in maintaining health is preservation of balance, described in each paradigm as homeostasis or balance of yin and yang, respectively. In the process of allostasis, the organism invokes physiological changes mediated by broadly acting regulatory systems in response to a stressor that threatens (or is perceived as threatening) homeostasis. The TCM theory of yin and yang describes this same phenomenon. However, instead of referring to the mediators of homeostatic regulation as glucocorticoids, serotonin or catecholamines, TCM mediators are described by terminology such as qi, blood and essence.

Both models allow for a period during which an individual is able to accommodate for stressors. The breakdown of homeostasis involves several factors. One key factor is an individual's underlying constitution. Allostasis describes inherited genetic traits and early life events as conferring stress resilience to an individual. In TCM, this is referred to as a person's jing (19-21). These references to a person's innate reserve explain the common clinical experience that individuals differ in stress adaptabilities and susceptibilities. Regarding functional somatic syndromes, this underlying vulnerability accounts for individual tendencies to develop adverse outcomes from stressors that most individuals are otherwise able to tolerate.

In conjunction with an individual's underlying constitution, the breakdown of homeostasis results from chronic, sustained stress, with or without punctuation by a severe, acute stressor. Patients with functional somatic syndromes often convey a long history of repeated life stressors during which most symptoms are self-limiting or relatively quiescent. This may represent initial adaptive responses of homeostatic mechanisms. However, either after an acutely stressful event or after several years of stressors, symptoms seem to suddenly arise and persist thereafter. This is consistent with both models in which, after a critical degree of stress, compensatory mechanisms are overloaded and thereafter fail to function optimally, ultimately leading to symptoms. In this way, the concept of allostatic load can be considered similar to the patterns of dysregulation in TCM.

Rather than affecting only one peripheral target, both ancient and modern models purport that pathological stressors systemically disturb the mind-brain-body continuum. (A pathological stressor, in this context, refers to a perturbation that is severe or sustained enough to result in allostatic load.) 
In TCM, stressors induce imbalances leading to an underlying pattern of dysregulation which manifest with multisystemic symptomatology. Within the model of allostatic load, stressors affect broadly acting regulating mechanisms, resulting in a similar multisystemic presentation. This conceptualization better explains why the majority of patients with functional somatic syndromes, regardless of the specific disorder for which they seek medical care, will present symptoms from multiple systems. Further, many present complex symptoms such as fatigue, lethargy, low resilience, sleep disturbance and mood disturbance, which are difficult to explain by a specific organ dysfunction. These systemic symptoms are easily explained by a complex interplay of alterations in ascending monoaminergic systems (e.g., depletion of serotoninergic mechanisms and upregulation of noradrenergic systems), tonic and phasic alterations of autonomic nervous system activity (e.g., low cardiovagal tone, increased sympathetic nervous system reactivity), alterations in HPA axis tone and responsiveness and tonic and phasic pain modulation systems (38-41).

\section{Limitations}

As intriguing as the comparison between the ancient TCM paradigm and the emerging modern neurobiological models may be, they are simply comparisons at this point. The similarities are most striking in the TCM pattern diagnosis, where specific somatic patterns are associated with specific alterations of emotions. Other aspects of the TCM paradigm, such as the concept of $q i$ and the meridians, have not been addressed in our discussion and may be more difficult to address from a Western scientific perspective. Finally, the apparent similarities between TCM pattern classification and distinct mind-brain-body states will need to be verified in future hypothesis-driven mechanistic studies.

\section{Future Directions}

The correlations between TCM, allostasis/allostatic load and EMS models offer exciting opportunities to scientifically test novel theories on the pathogenesis of the functional somatic syndromes and to investigate biomedical explanations underlying ancient TCM terms and concepts. For example, in the case of IBS, current diagnosis under the Rome criteria focuses solely on bowel symptoms (42). However, in clinical practice most IBS patients present significant extra-intestinal symptomatology. In fact, clinical evidence suggests that normalization of bowel habits alone by anti-diarrheals or pro-motility drugs is unlikely to produce large improvements in global endpoints when compared to placebo $(33,43)$.

Further, the present group has demonstrated that both mental and physical component scores for HRQoL in IBS are primarily determined by extra-intestinal symptoms rather than conventionally-elicited bowel symptoms (29). Rather than conventionally subgrouping IBS patients on the basis of bowel habits alone (e.g., constipation- versus diarrhea-pre- dominant), TCM broadly subgroups patients into deficiency (i.e., yin) or excess (i.e., yang) types based on extra-intestinal symptoms such as fatigue versus hyperactivity or weak stress resilience versus stress-induced overstimulation. The present group has hypothesized that this TCM approach potentially offers novel and scientifically testable subgroups of IBS representing differential dysfunction (i.e., hypofunctioning in yin types and hyper-responsiveness in yang types) of allostasis and EMS output profiles. Specifically, we have begun preliminary physiological studies comparing basal and stressinduced autonomic differences in IBS patients to determine if our hypothesis is supported. We believe integrative EastWest approaches such as this represent a sound first step towards scientifically validating the ancient theories of TCM while throwing light on central physiological derangements underlying the functional somatic syndromes.

\section{Conclusions}

The functional somatic syndromes pose a challenge to modern medicine, because they are primarily symptom-based, multisystemic in presentation and probably involve complex mind-brain-body interactions. They represent a group of disorders that cannot be easily classified under the conventional biomedical model which is primarily focused on specific diseases defined by structural, and often microscopic, singlevariable parameters confined to a particular organ. The challenge at hand is to find novel approaches that incorporate a macroscopic perspective, taking into account the toll of acute and chronic traumas, physical and emotional stressors and the complex interaction between the mind, brain and body.

The emerging biomedical models of allostasis/allostatic load and the EMS show striking similarities to TCM in conceptualization of the functional somatic disorders and their underlying pathogenesis. The convergence of these models may provide new insight into scientifically verifiable diagnostic and therapeutic approaches for these common disorders. From a broader perspective, further exploration of the links between these models will undoubtedly bridge the gap in our understanding of the relationships between mind and body, ancient wisdom and modern science, and Eastern and Western approaches to health and disease.

\section{References}

1. Barksy AJ, Borus JF. Functional somatic syndromes. Ann Intern Med 1999;130:910-21.

2. Aaron LA, Buchwald D. A review of the evidence for overlap among unexplained clinical conditions. Ann Intern Med 2001;134:868-81.

3. Wessely S. Chronic fatigue: symptom and syndrome. Ann Intern Med 2001;134:838-43.

4. Sharpe M, Carson A. 'Unexplained' somatic symptoms, functional syndromes, and somatization: do we need a paradigm shift? Ann Intern Med 2001;134:926-30.

5. Kroenke K, Harris L. Symptoms research: a fertile field. Ann Intern Med 2001;134:801-2.

6. Aronowitz RA. When do symptoms become a disease? Ann Intern Med 2001;134:803-8. 
7. Katon W, Sullivan M, Walker E. Medical symptoms without identified pathology: relationship to psychiatric disorders, childhood and adult trauma, and personality traits. Ann Intern Med 2001;134:917-25.

8. Wolfe F, Smythe HA, Yunus MB, Bennett RM, Bombardier C, Goldenberg DL, et al. The American College of Rheumatology 1990 criteria for the classification of fibromyalgia, a report of the Multicenter Criteria Committee. Arthritis Rheum 1990;33:160-72.

9. Yunus MB. A comprehensive medical evaluation of patients with fibromyalgia syndrome. Rheum Dis Clin North Am 2002;28:201-17.

10. Wessely S, Nimnuan C, Sharpe M. Functional somatic syndromes: one or many? Lancet 1999;354:936-9.

11. Henningsen P, Zimmermann T, Sattel H. Medically unexplained physical symptoms, anxiety, and depression: a meta-analytic review. Psychosom Med 2003;65:528-33.

12. Eisenberg DM, Davis RB, Ettner SL, Appel S, Wilkey S, Van Rompay M, et al. Trends in alternative medicine use in the United States, 1990 1997: results of a follow-up national survey. JAMA 1998;280:1569-75.

13. Smart HL, Mayberry JF, Atkinson M. Alternative medicine consultations and remedies in patients with the irritable bowel syndrome. Gut 1986;27:826-8.

14. McEwen BS. Interacting mediators of allostasis and allostatic load: towards an understanding of resilience in aging. Metabolism 2003;52: $10-16$.

15. McEwen BS, Seeman T. Protective and damaging effects of mediators of stress: elaborating and testing the concepts of allostasis and allostatic load. Ann NY Acad Sci 1999;896:30-47.

16. Holstege G, Bandler R, Saper CB. The emotional motor system. In: Holstege G, Bandler R, Saper CB, editors. The Emotional Motor System. Amsterdam: Elsevier: 1996; 3-6.

17. Holstege G. The emotional motor system. Eur J Morphol 1992;30:6779.

18. McEwen BS. The end of stress as we know it. Washington D.C.: Joseph Henry Press: 2002.

19. Kaptchuk TJ. The web that has no weaver: understanding Chinese medicine. Chicago, IL: Congdon \& Weed, Inc.: 1983.

20. Maciocia G. The foundations of Chinese medicine: a comprehensive text for acupuncturists and herbalists. New York, NY: Churchill Livingstone: 1989

21. Maciocia G. The practice of Chinese medicine: the treatment of diseases with Acupuncture and Chinese herbs. New York, NY: Churchill Livingstone: 1994

22. Moynihan R, Smith R. Too much medicine? Almost certainly. BMJ 2002;324:859-60.

23. Moynihan R. Drug firms hype disease as sales ploy, industry chief claims. BMJ 2002;324:867.

24. Moynihan R, Heath I, Henry D. Selling sickness: the pharmaceutical industry and disease mongering. $B M J$ 2002;324:886-90.

25. Lièvre M. Alosetron for irritable bowel syndrome. BMJ 2002;325:5556.

26. Moynihan R. FDA advisers warn of more deaths if drug is relaunched. BMJ 2002;325:561.
27. Moynihan R. Alosetron: a case study in regulatory capture, or a victory for patients' rights. $B M J 2002 ; 325: 592-5$.

28. Gralnek IM, Hays RD, Kilbourne A, Naliboff B, Mayer EA. The impact of irritable bowel syndrome on health-related quality of life. Gastroenterology 2000;119:654-60.

29. Spiegel BMR, Gralnek IM, Mayer EA, Bolus R, Dulai GS, Chang L, et al. Clinical determinants of health-related quality of life in irritable bowel syndrome. Arch Intern Med 2004. (In Press)

30. Mayer EA. The neurobiology of stress and gastrointestinal disease. Gut 2000;47:861-9.

31. Manu P (editor). Functional Somatic Syndromes: etiology, diagnosis, and treatment. Cambridge, UK: Cambridge University Press: 1998.

32. Hudson JI, Pope HGJ. Fibromyalgia and psychopathology: is fibromyalgia a form of 'affective spectrum disorder'? J Rheumatol Suppl 1989;19:15-22.

33. Jailwala J, Imperiale TF, Kroenke K. Pharmacologic treatment of the irritable bowel syndrome: a systematic review of randomized, controlled trials. Ann Intern Med 2000;133:136-47.

34. Klein KB. Controlled treatment trials in the irritable bowel syndrome: a critique. Gastroenterology 1988;95:232-41.

35. Tan S, Tillisch K, Bolus R, Olivas TI, Naliboff BD, Chang L, et al. Subgrouping of irritable bowel syndrome (IBS) patients using traditional Chinese medicine (TCM) symptom classification. (Forthcoming)

36. McEwen BS. Protective and damaging effects of stress mediators. $N$ Engl J Med 1998;338:171-9.

37. LeDoux JE. The emotional brain: the mysterious underpinnings of emotional life. New York: Simon \& Schuster: 1996.

38. Vgontzas AN, Chrousos GP. Sleep, the hypothalamic-pituitary-adrenal axis, and cytokines: multiple interactions and disturbances in sleep disorders. Endocrinol Metab Clin North Am 2002;31:15-36.

39. Patarca-Montero R, Antoni M, Fletcher MA, Klimas NG. Cytokine and other immunologic markers in chronic fatigue syndrome and their relation to neuropsychological factors. Appl Neuropsychol 2001;8:51-64.

40. Elenkov IJ, Chrousos GP. Stress hormones, Th1/Th2 patterns, pro/ anti-inflammatory cytokines and susceptibility to disease. Trends Endocrinol Metab 1999;10:359-68.

41. Madden KS. Catecholamines, sympathetic nerves and immunity. In: Ader R, Felten D, Cohen N, editors. Psychoneuroimmunology. New York, NY: Academic Press: 2001, 197-216.

42. Drossman DA, Corazziari E, Talley NJ, Thompson WG, Whitehead WE. Drossman DA, Corazziari E, Talley NJ, Thompson WG, Whitehead WE, editors. ROME II. The functional gastrointestinal disorders. Diagnosis, pathophysiology and treatment: a multinational consensus. McLean, VA: Degnon Associates: 2000.

43. Fennerty MB. Traditional therapies for irritable bowel syndrome: an evidence-based appraisal. Rev Gastroenterol Disord 2003;3:S18-24.

44. Mayer EA, Naliboff BD, Chang L, Coutinho SV. Stress and the gastrointestinal tract V. Stress and irritable bowel syndrome. Am J Physiol Gastrointest Liver Physiol 2001;280:G519-24.

45. Mayer EA, Collins SM. Evolving pathophysiologic models of functional gastrointestinal disorders. Gastroenterology 2002;122:2032-48.

Received December 19, 2003; accepted February 24, 2004 


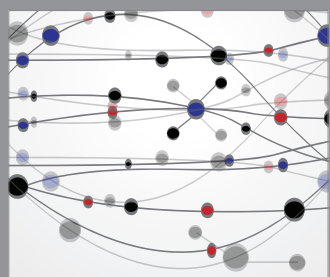

The Scientific World Journal
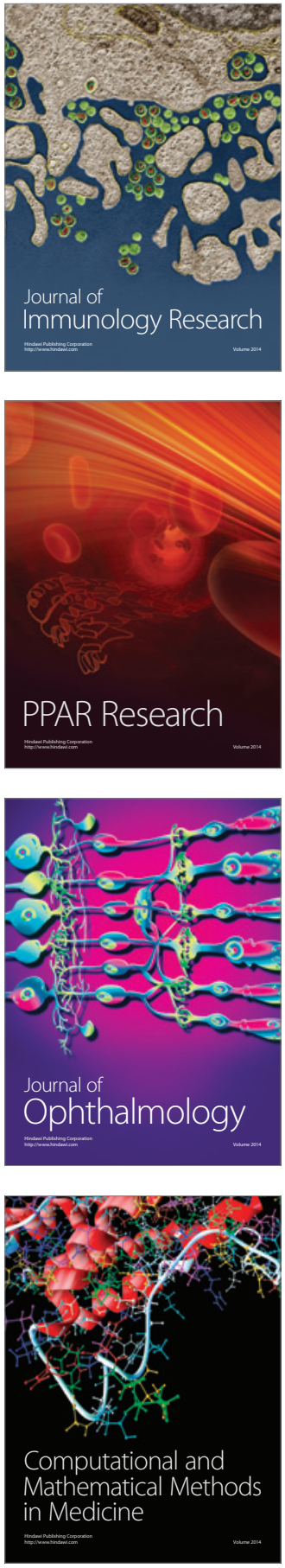

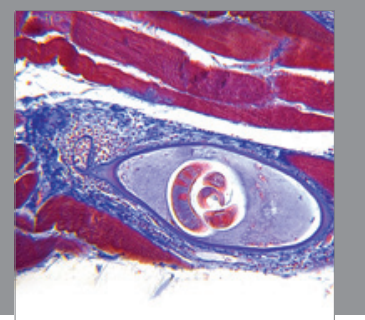

Gastroenterology

Research and Practice
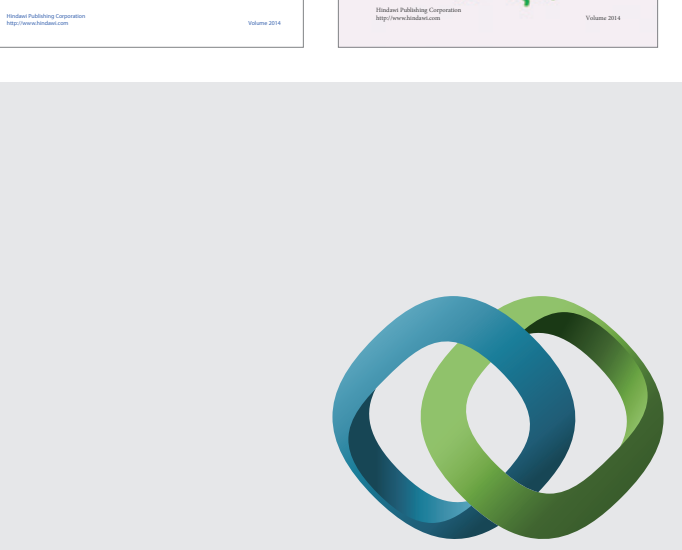

\section{Hindawi}

Submit your manuscripts at

http://www.hindawi.com
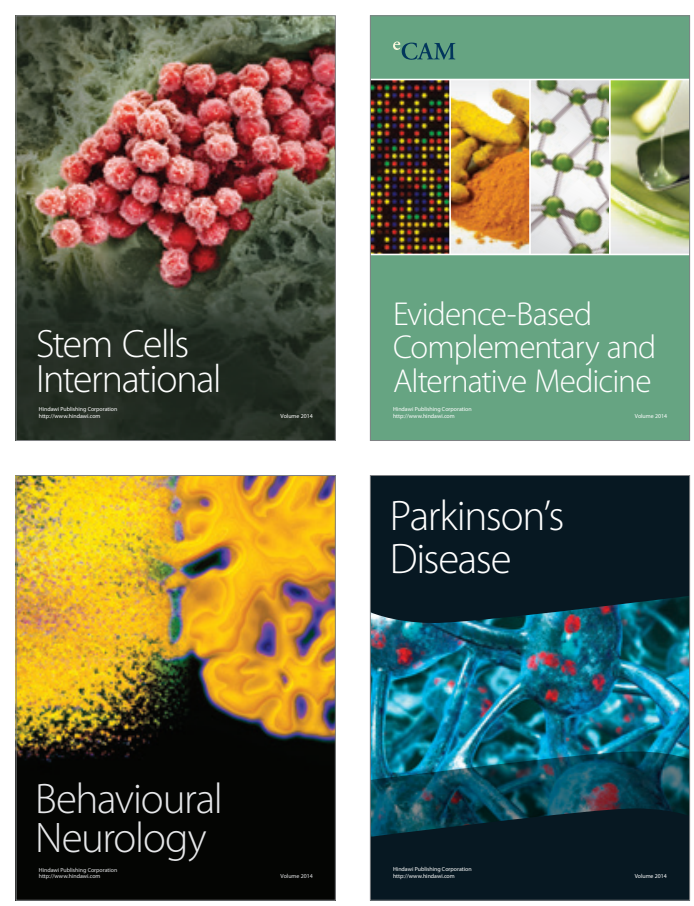

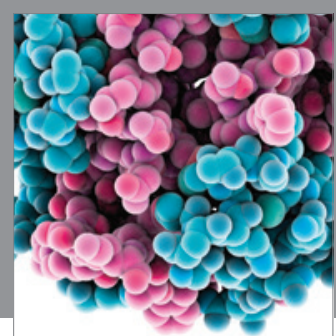

Journal of
Diabetes Research

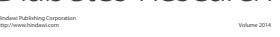

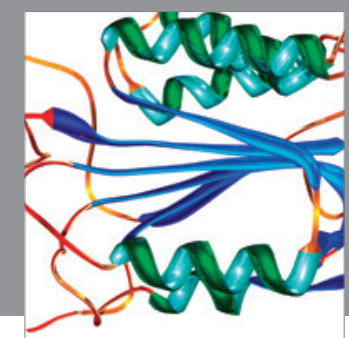

Disease Markers
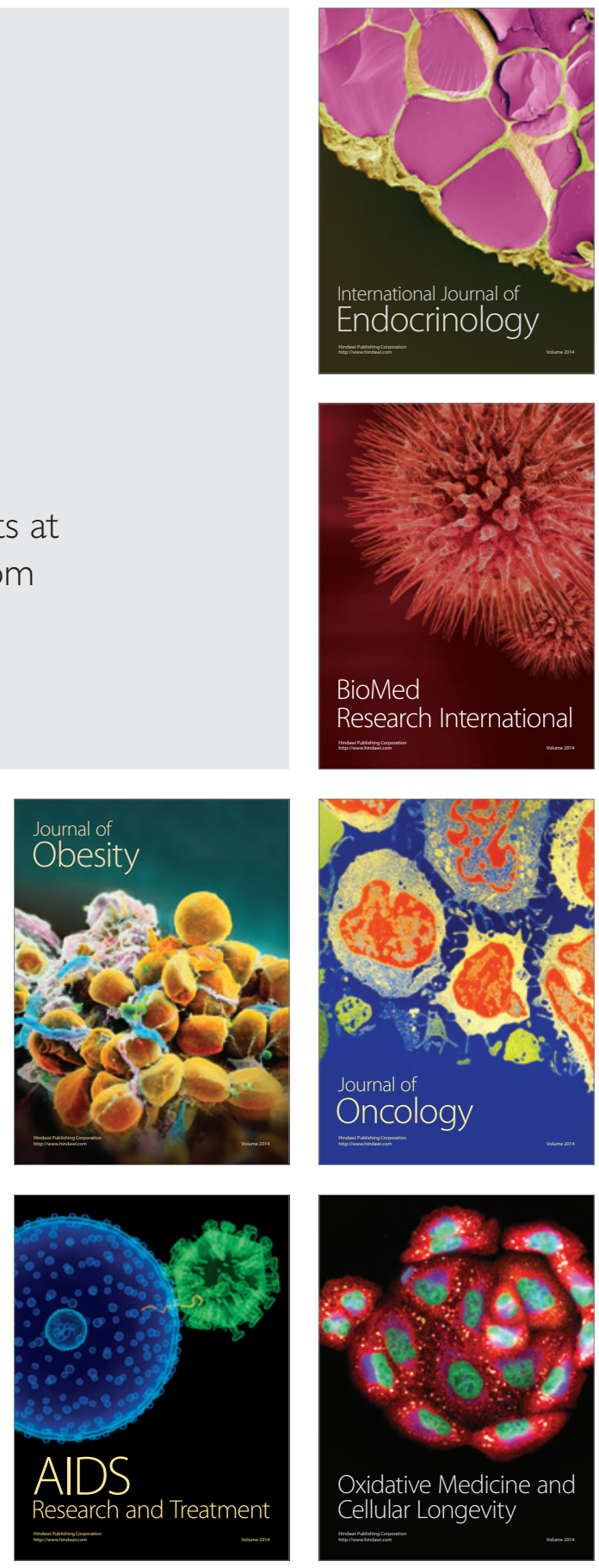